\title{
Factorisation of recursion operators of some Lagrangian systems
}

Dmitry K. Demskoi

To cite this article: Dmitry K. Demskoi (2017) Factorisation of recursion operators of some Lagrangian systems, Journal of Nonlinear Mathematical Physics 24:3, 368-378, DOI: https://doi.org/10.1080/14029251.2017.1341699

To link to this article: https://doi.org/10.1080/14029251.2017.1341699

Published online: 04 January 2021 


\title{
Factorisation of recursion operators of some Lagrangian systems
}

\author{
Dmitry K. Demskoi \\ Charles Sturt University \\ School of Computing and Mathematics, Charles Sturt University \\ New South Wales 2678, Australia \\ ddemskoy@csu.edu.au
}

Received 8 November 2016

Accepted 11 April 2017

\begin{abstract}
We observe that recursion operator of an $S$-integrable hyperbolic equation that degenerates into a Liouvile-type equation admits a particular factorisation. This observation simplifies the construction of such operators. We use it to find a new quasi-local recursion operator for a triplet of scalar fields. The method is also illustrated with examples of the sinh-Gordon, the Tzitzeica and the Lund-Regge equations.
\end{abstract}

Keywords: Recursion operator; first integral; generalised symmetry.

\section{Introduction}

We consider a system that belongs to a family of $S$-integrable systems with the Lagrangians of the form

$$
L=g_{i j}(u) u_{x}^{i} u_{t}^{j}+f(a, u),
$$

where $a=$ const and $g_{i j}$ is the metric tensor of a (pseudo)Riemannian space with local coordinates $u=\left(u^{1}, \ldots, u^{n}\right)$. Throughout the article we assume summation over repeated indices. The list of Lagrangians (1.1) in a three-dimensional reducible space such that the respective field equations are integrable was presented in [4] (see also [3]).

Probably the most interesting system from the list is obtained from the Lagrangian

$$
L=\frac{1}{2} u_{t} u_{x}+\psi\left(v_{x} w_{t}+w_{x} v_{t}\right)+v e^{u}+a w e^{-u},
$$

where $\left(u^{1}, u^{2}, u^{3}\right)=(u, v, w)$,

$$
\psi=(v w+c)^{-1}
$$

and $c \neq 0$ is a constant. Let us indicate some of the known facts about this system. Its integrability was established in [5] (see also [3]) by constructing a Lax representation in terms of $3 \times 3$ matrices. Remarkably for all other systems in the list, the Lax representations are given in terms of larger matrices. The system possesses a second order symmetry which is related to the Yajima-Oikawa system [22] by a Miura-type transformation [2]. Further, it was shown in [2] that on setting $a=$ 0 the system degenerates into a Liouville-type system, in which case the integrals can be easily derived by using system's generalised symmetry. One of the missing, yet important, structures is a recursion operator (see [19] for definition and properties). There are a few approaches towards 
solving this problem. For the system in question, a recursion operator can be constructed from the recursion operator of the Yajima-Oikawa equation [13]. This, however, would entail a tedious process of inverting matrix differential operators. On the other hand, the procedure of [10], or other methods (see e.g. [16], [15], [12]) can be used to derive a recursion operator from the known Lax representation. This method is best suited for Lax pairs of a particular form, e.g. Gelfand-Dikii systems. However, in the present case the simplest approach seems neither of the mentioned two, but the one which exploits the Lagrangian form of the system in question and properties of the related Liouville-type system [23]. The method presented below is applicable to other systems as well, so on the one hand it is convenient to keep the presentation general enough and on the other, for the sake of clarity, we illustrate it with a simpler example of the equation

$$
u_{t x}=e^{-2 u}+a e^{2 u} \text {, }
$$

which is equivalent to the sinh-Gordon equation. Equation (1.4) degenerates into the Liouville equation

$$
u_{t x}=e^{-2 u}
$$

on setting $a=0$. Obviously equations (1.4) and (1.5) have Lagrangians of the form $L=u_{t} u_{x} / 2+f(a, u)$, which is a particular instance of (1.1).

The Liouville equation, known to be $C$-integrable, has a curious property that its symmetry algebra contains the symmetry algebras of the sinh-Gordon and the Tzitzeica

$$
u_{t x}=e^{-2 u}+a e^{u}
$$

equations. This property follows from the fact that generalised symmetries of these equations do not depend on constant $a$, and implies that their recursion operators are, at the same time, the recursion operators of the Liouville equation. Thus our objective is to develop a procedure for constructing recursion operators of the Liouville-type equations and specifying them for the related $S$-integrable equations. In the context of our illustrative example, we would like to construct a general recursion operator of the Liouville equation and then specify it for the sinh-Gordon and Tzitzeica equations. This is done in the following section. In the last section we consider our main example - the system with Lagrangian (1.2).

\section{Recursion operators for Liouville-type systems}

We start with a derivation of generalised symmetries from the integrals of the Liouville-type equations (or systems). A general method for this was presented in [6]. In a particular case of Lagrangian systems, it takes much simpler and more algorithmic form. The simplification is due to the linearised equation being self-adjoint (see formula (2.9) below).

Consider a hyperbolic equation (or system) in Lagrangian form

$$
\mathscr{E}_{u^{k}}(L)=0,
$$

where

$$
\mathscr{E}_{u^{k}}=\frac{\partial}{\partial u^{k}}-\partial_{t} \frac{\partial}{\partial u_{t}^{k}}-\partial_{x} \frac{\partial}{\partial u_{x}^{k}}+\partial_{t}^{2} \frac{\partial}{\partial u_{t t}^{k}}+\partial_{x}^{2} \frac{\partial}{\partial u_{x x}^{k}}-\ldots
$$

is Euler's operator. In order to avoid confusion with using different types of brackets, we adhere to the convention: an operator followed by a scalar expression in parentheses stands for the result of 
application of the operator to the expression. We will omit the subscript $u^{k}$ when we do not want to single out a particular equation. Note that (2.1) can be written in terms of the Fréchet derivative

$$
L_{*}=\frac{\partial L}{\partial u^{i}}+\frac{\partial L}{\partial u_{x}^{i}} \partial_{x}+\frac{\partial L}{\partial u_{t}^{i}} \partial_{t}+\frac{\partial L}{\partial u_{x x}^{i}} \partial_{x}^{2}+\frac{\partial L}{\partial u_{t t}^{i}} \partial_{t}^{2}+\ldots
$$

as

$$
L_{*}^{\dagger}(1)=0,
$$

where symbol ${ }^{\dagger}$ indicates passing to the formal adjoint operator.

Suppose

$$
\omega=\omega\left(u, u_{x}, u_{x x}, \ldots\right) .
$$

is the simplest $x$-integral of equation (2.1), i.e. the integral of the lowest order that satisfies the relation

$$
D_{t}(\omega)=0
$$

on solutions of (2.1). Here $D_{t}$ is the operator of the total derivative with respect to $t$ calculated on solutions of (2.1). The latter implies that we must eliminate all mixed derivatives by means of (2.1). Similarly, the operator of the $x$-derivative followed by elimination of mixed derivatives will be denoted as $D_{x}$.

Definition. We call system of equations (2.1) a Liouville-type system if it possesses $2 n$ independent non-trivial integrals (see e.g. [24], [23]).

Without elimination of the mixed derivatives, relation (2.3) can be usually cast into the form

$$
\partial_{t}(\omega)=\mathscr{A} \mathscr{E}(L)
$$

where $\mathscr{A}$ is a linear differential operator which we call an integrating factor corresponding to integral $\omega$. For example, for the simplest $x$-integral

$$
\omega=u_{x x}+u_{x}^{2},
$$

of the Liouville equation, relation (2.4) is given by

$$
\partial_{t}\left(u_{x x}+u_{x}^{2}\right)=\left(\partial_{x}+2 u_{x}\right) \mathscr{E}\left(-u_{t} u_{x} / 2-1 / 2 e^{-2 u}\right) .
$$

If we assume that $\mathscr{A}$ has the form

$$
\mathscr{A}=\sum_{i} a_{i} \partial_{x}^{i}
$$

then from the well-know properties of the Fréchet derivative, it follows that

$$
(\mathscr{A}(h))_{*}=\mathscr{A} h_{*}+\mathscr{A} *[h],
$$


where

$$
\mathscr{A}_{*}[h]=\sum_{i} \partial_{x}^{i}(h)\left(a_{i}\right)_{*}
$$

Applying the Fréchet derivative to (2.4), we obtain

$$
\partial_{t} \circ \omega_{*}=\mathscr{A} \circ(\mathscr{E}(L))_{*}+\mathscr{A}_{*}[\mathscr{E}(L)] .
$$

The latter, being calculated on solutions of (2.1), takes the form

$$
D_{t} \circ \omega_{*}=\mathscr{A} \circ(\mathscr{E}(L))_{*} .
$$

This relation allows one to calculate the integrals of (2.1) from symmetries of the same equation. Indeed, (2.6) shows that any operator of the form

$$
\mathscr{L}=\mathscr{K} \omega_{*},
$$

where $\mathscr{K}=\mathscr{K}\left(\omega, \omega_{x}, \ldots\right)$ is an operator depending on $x$-integrals of (2.1), transforms symmetries of (2.1) to the integrals of the same equation.

For example, for the Liouville equation the operator possessing such a property is given by

$$
\mathscr{L}=\mathscr{K}\left(D_{x}^{2}+2 u_{x} D_{x}\right) .
$$

Setting

$$
\mathscr{K}=D_{x}^{-1}
$$

we get

$$
\mathscr{L}=D_{x}+2 D_{x}^{-1} u_{x} D_{x}=D_{x}+2 u_{x}-2 D_{x}^{-1} u_{x x} .
$$

It is easy to check that if we apply this operator to the translational symmetry $u_{x}$, we obtain integral (2.5).

Further, calculating the formal adjoint of (2.6) and taking into account that

$$
(\mathscr{E}(L))_{*}^{\dagger}=(\mathscr{E}(L))_{*},
$$

we obtain

$$
-\omega_{*}^{\dagger} D_{t}=(\mathscr{E}(L))_{*} \mathscr{A}^{\dagger} .
$$

The latter shows that operator $\mathscr{A}^{\dagger}$ maps $x$-integrals of (2.1) into its symmetries. Now we can summarise the previous lines of reasoning in the following

Proposition. Given a hyperbolic equation in Lagrangian form (2.1) along with its simplest $x$ integral $\omega$ such that representation (2.4) holds. Then, this equation possesses a recursion operator of the form

$$
\mathscr{R}=\mathscr{A}^{\dagger} \mathscr{K} \omega_{*},
$$

where $\mathscr{K}$ is a linear operator that depends on integral $\omega$ (and possibly its derivatives). 
For the Liouville equation we have

$$
\mathscr{A}^{\dagger}=\left(D_{x}+2 u_{x}\right)^{\dagger}=2 u_{x}-D_{x}
$$

hence the symmetries ${ }^{\mathrm{a}}$ of the Liouville equation equation can be represented as

$$
u_{\tau}=\left(2 u_{x}-D_{x}\right)(f)
$$

where $f=f\left(\omega, \omega_{x}, \ldots\right)$ is a scalar function, and any operator of the form

$$
\mathscr{R}=\left(D_{x}-2 u_{x}\right) \mathscr{K}\left(D_{x}^{2}+2 u_{x} D_{x}\right)
$$

is a recursion operator of (1.5).

Since the symmetry algebras of the sinh-Gordon and the Tzitzeica equations are sub-algebras of the one of the Liouville equation, the recursion operators of (1.4) and (1.6) must have form (2.12) where $\mathscr{K}$ is an operator with coefficients depending on $\omega$ and possibly its derivatives.

To ensure that (2.12) is the recursion operator of the sinh-Gordon we need to specify $\mathscr{K}$ such that (2.12) also satisfies the commutator equation

$$
\left[D_{t}+2 D_{x}^{-1} \circ\left(e^{-2 u}-a e^{2 u}\right), \mathscr{R}\right]=0
$$

on solutions of the sinh-Gordon equation. For the sinh-Gordon equation, operator $\mathscr{K}$ can be easily found: one can verify that it is given by formula (2.8).

To obtain the recursion operator of the Tzitzeica equation we must find $\mathscr{K}$ such that

$$
\left[D_{t}+D_{x}^{-1} \circ\left(2 e^{-2 u}-a e^{u}\right), \mathscr{R}\right]=0 .
$$

A straightforward search for operator $\mathscr{K}$ in this case is more difficult since the answer is a third order nonlocal operator:

$$
\mathscr{K}=D_{x}^{-1}\left(D_{x}^{2}-\omega\right) D_{x}\left(D_{x}^{2}-\omega\right) D_{x}^{-1}
$$

The relation of operators (2.8) and (2.14) to Hamiltonian formalism is explained in the remaining part of this section.

\section{Hamiltonian interpretation}

The missing ingredient in formula (2.11) is operator $\mathscr{K}$ which only depends on the integrals. The integrals, in turn, are known [21,24] to generate Miura-type transformations for the symmetries of Liouville-type equations. Thus it is natural to investigate whether there is a connection between operator $\mathscr{K}$ and one of the structures related to the modified hierarchies. This turns out to be the case, namely operator $\mathscr{K}$ is a symplectic operator of the modified hierarchy.

$\overline{{ }^{\mathrm{a}} \text { For simplicity we only }}$ consider translation-invariant symmetries. 
Suppose equation (2.1) admits a hierarchy of generalised symmetries

$$
u_{\tau_{i}}=F_{i}\left(u, u_{x}, \ldots\right),
$$

where $\tau_{-1}=t$, generated by a recursion operator $\mathscr{R}: F_{i+1}=\mathscr{R}\left(F_{i}\right)$. We assume that $F_{i}$ for $i>-1$ do not depend on constant $a$. The hierarchy of modified equations

$$
\omega_{\tau_{i}}=G_{i}\left(\omega, \omega_{x}, \ldots\right)
$$

is related to (2.15) by means of integral (2.2), interpreted as a differential substitution. Note that $G_{-1}$ is a non-local expression. We denote as $\mathfrak{R}$ the recursion operator of hierarchy (2.16). It is well known [7] that $\mathscr{R}$ and $\mathfrak{R}$ are related by the formula $\omega_{*} \mathscr{R}=\mathfrak{R} \omega_{*}$. Further, we assume that (2.15) has a Hamiltonian form with

$$
F_{i}=\mathscr{J} \mathscr{E}_{u}\left(H_{i}\right), H_{-1}=f,
$$

where $\mathscr{J}$ is an implectic (Hamiltonian) operator and $H_{i}$ are the respective Hamiltonian densities. It was indicated in [18] that the operator with components

$$
\mathscr{M}_{i j}=g_{i j} \partial_{x}+g_{i l} \Gamma_{j k}^{l} u_{x}^{k}
$$

where $\Gamma_{j k}^{l}$ are the coefficients of the Levi-Civita connection for the metric $g_{i j}$, is symplectic. Hence in order to find operator $\mathscr{J}$ explicitly, one has to invert operator $\mathscr{M}$. Clearly operator $\mathscr{J}$ is nonlocal and can be quite tedious to calculate. However, as it is shown in the following calculations we do not need to know the explicit form of $\mathscr{J}$ if we are interested in calculating the recursion operator of (2.1) or (2.15).

The fact that hierarchy (2.15) is obtained by making a differential substitution implies that it can be re-written in the form

$$
u_{\tau_{i}}=\left.\mathscr{J} \omega_{*}^{\dagger} \mathscr{E}_{\omega}\left(H_{i}\right)\right|_{\omega=\omega\left(u, u_{x}, \ldots\right)}
$$

while (2.16) can be written as

$$
\omega_{\tau_{i}}=\omega_{*} \mathscr{J} \omega_{*}^{\dagger} \mathscr{E} \omega\left(H_{i}\right)
$$

The operator $\omega_{*} \mathscr{J} \omega_{*}^{\dagger}$ is usually referred to as the second Hamiltonian operator of hierarchy (2.16). Comparing (2.4) and (2.19) for $i=-1$ we find that $\mathscr{A}=\omega_{*} \mathscr{J}$. Operator $\mathscr{A}$ is always local hence much easier to handle in calculations compared to operator $\mathscr{J}$.

Now suppose we also know a symplectic operator $\mathscr{S}$ for hierarchy (2.16) such that its recursion operator is factorisable as

$$
\mathfrak{R}=\omega_{*} \mathscr{J} \omega_{*}^{\dagger} \mathscr{S} .
$$

It is well known (see e.g. $[8,19]$ ) that the formal adjoint of $\mathfrak{R}$ relates the members of the hierarchy of conserved covariants (cosymmetries):

$$
\mathscr{E}_{\omega}\left(H_{i+1}\right)=\mathfrak{R}^{\dagger} \mathscr{E}_{\omega}\left(H_{i}\right) .
$$

On the other hand, relation (2.18) implies

$$
\mathscr{R} \mathscr{J} \omega_{*}^{\dagger} \mathscr{E}_{\omega}\left(H_{i}\right)=F_{i+1}=\mathscr{J} \omega_{*}^{\dagger} \mathscr{E} \omega\left(H_{i+1}\right)=\mathscr{J} \omega_{*}^{\dagger} \mathfrak{R}^{\dagger} \mathscr{E}_{\omega}\left(H_{i}\right)
$$


hence

$$
\mathscr{R} \mathscr{J} \omega_{*}^{\dagger}=\mathscr{J} \omega_{*}^{\dagger} \mathfrak{R}^{\dagger} .
$$

Substituting (2.20) in (2.21), we obtain

$$
\mathscr{R}=\mathscr{J} \omega_{*}^{\dagger} \mathscr{S} \omega_{*}=\mathscr{A}^{\dagger} \mathscr{S} \omega_{*} .
$$

Comparing formulae (2.11) and (2.22) we find that operators $\mathscr{S}$ and $\mathscr{K}$ coincide thus we conclude that a symplectic operator for hierarchy (2.16) plays a role of operator $\mathscr{K}$ in formula (2.11). We summarise the last part of this section in the following

Proposition. Given a hyperbolic equation in Lagrangian form (2.1). If this equation

a) has an infinite hierarchy of symmetries (2.15);

b) degenerates (e.g. by assigning a particular value to a parameter) into a Liouville-type equation such that the degenerate equation possesses the same hierarchy of symmetries (2.15);

c) gives rise (through an integral of the degenerate equation) to the modified hierarchy that has a symplectic operator $\mathscr{S}$;

then, (2.1) has a recursion operator of the form (2.22).

Remarks. The main obstacle to applicability of this proposition is condition b). Namely, there are numerous examples of hyperbolic equations and systems whose symmetries depend on a parameter that is used to obtain the degenerate equation. On the other hand, condition b) can be weakened, i.e. rather than requiring the equation to be of the Liouville type, we may demand existence of less than $n$ integrals. However, this sort of examples usually lead to triangular systems (e.g. system (6) in [5]).

One can recognise that the operator given by formula (2.14) is a symplectic operator for the Sawada-Kotera hierarchy which is related to the Tzitzeica hierarchy through substitution (2.5).

\section{Complex sine-Gordon system}

Before we proceed with our main example, we consider another system with a Lagrangian of the form (1.1), namely the complex sine-Gordon also known as the Lund-Regge system [14]:

$$
v_{t x}=\psi w v_{t} v_{x}+a v \psi^{-1}, w_{t x}=\psi v w_{t} w_{x}+a w \psi^{-1},
$$

where $\psi$ is given by (1.3) and $a, c=$ const. This system gives yet another example where the method presented here can be used. The calculation is slightly more involved, compared to the sinh-Gordon equation. In the formulae below it suffices to list only the key ingredients, e.g. Lagrangian densities, integrals, etc, while the recursion operator is readily calculated by substituting the ingredients in formula (2.11).

System (3.1) is derived from the Lagrangian

$$
L=\psi v_{t} w_{x}+a v w
$$

The simplest generalised symmetry of (3.1) belongs to the list of non-linear Shrödinger type systems [17] and is given by

$$
\begin{gathered}
v_{\tau}=v_{x x}-2 \nu \psi v_{x} w_{x}, \quad w_{\tau}=-w_{x x}+2 w \psi w_{x} v_{x} . \\
\text { Co-published by Atlantis Press and Taylor \& Francis } \\
\text { Copyright: the authors }
\end{gathered}
$$


On setting $a=0$ system (3.1) degenerates into the system

$$
v_{t x}=\psi w v_{t} v_{x}, w_{t x}=\psi v w_{t} w_{x}
$$

which was identified as a Liouville-type system in [6]. Importantly, system (3.2) does not depend on constant $a$, hence formula (2.11) can be used to construct the recursion operator of (3.1).

The simplest integral $\omega=(\rho, \theta)^{T}$ of (3.3) is given by

$$
\rho=v_{x} w_{x} \psi, \quad \theta=\frac{w_{x x}}{w_{x}}-w v_{x} \psi
$$

This integral gives rise to a differential substitution relating (3.2) with the well-known Kaup-Broer system $[1,11]$

$$
\rho_{\tau}=\rho_{x x}-2(\rho \theta)_{x}, \quad \theta_{\tau}=-\theta_{x x}-\left(\theta^{2}-2 \rho\right)_{x}
$$

which is known to have the symplectic operator

$$
\mathscr{K}=\left(\begin{array}{cc}
0 & D_{x}^{-1} \\
D_{x}^{-1} & 0
\end{array}\right)
$$

Operator $\omega_{*}$ reads

$$
\left(\begin{array}{l}
\rho \\
\theta
\end{array}\right)_{*}=\left(\begin{array}{cc}
0 & 0 \\
0 & w_{x}^{-1}
\end{array}\right) D_{x}^{2}+\left(\begin{array}{cc}
w_{x} \psi & v_{x} \psi \\
-w \psi & -w_{x x} w_{x}^{-2}
\end{array}\right) D_{x}-\psi^{2} v_{x}\left(\begin{array}{cc}
w w_{x} & v w_{x} \\
-w^{2} & c
\end{array}\right) .
$$

Further, the $t$-derivative of the integral can be written in form (2.4) with the operator

$$
\mathscr{A}=\left(\begin{array}{cc}
-v_{x} & -w_{x} \\
-D_{x} \circ\left(w_{x} \psi\right)^{-1}-v & w
\end{array}\right)
$$

hence

$$
\mathscr{A}^{\dagger}=\left(\begin{array}{cc}
-v_{x} & \left(w_{x} \psi\right)^{-1} D_{x}-v \\
-w_{x} & w
\end{array}\right)
$$

Substituting (3.8), (3.6) and (3.7) into (2.11) we obtain the recursion operator of (3.1) in the form

$$
\mathscr{R}=\left(\begin{array}{cc}
1 & 0 \\
0 & -1
\end{array}\right) D_{x}+\psi\left(\begin{array}{cc}
-v w_{x} & -2 v v_{x} \\
2 w w_{x} & w v_{x}
\end{array}\right)-\left(\begin{array}{c}
v \\
-w
\end{array}\right) D_{x}^{-1} \circ \mathscr{E}(\rho)-\left(\begin{array}{c}
v_{x} \\
w_{x}
\end{array}\right) D_{x}^{-1} \circ \mathscr{E}(\theta)
$$

Operator (3.9) was originally found by A.G. Meshkov who calculated it directly and also verified its hereditariness [16]. 


\section{Triplet of scalar fields}

Now consider our main example which is the system

$$
u_{x t}=v e^{u}-a w e^{-u}, \quad v_{x t}=\frac{a}{2} \psi^{-1} e^{-u}+\psi w v_{t} v_{x}, \quad w_{x t}=\frac{1}{2} \psi^{-1} e^{u}+\psi v w_{t} w_{x} .
$$

If we set $a=0$ the system becomes of the Liouville-type: the simplest integral $\omega=(\rho, \theta, \varphi)^{T}$ is given by [2]

$$
\rho=u_{x x}-\frac{1}{2} u_{x}^{2}-2 v_{x} w_{x} \psi, \quad \theta=v w_{x} \psi-\frac{v_{x x}}{v_{x}}-\frac{1}{2} u_{x}, \quad \varphi=\psi v_{x}\left(w_{x x}-w \psi v_{x} w_{x}-u_{x} w_{x}\right) .
$$

Further, the $t$-derivative of this integral can be written in the form (2.4) with the operator

$$
\begin{aligned}
\mathscr{A}=- & \frac{1}{2}\left(\begin{array}{ccc}
2 & 0 & 0 \\
0 & 0 & -\left(v_{x} \psi\right)^{-1} \\
0 & v_{x} & 0
\end{array}\right) D_{x} \\
& +\left(\begin{array}{ccc}
u_{x} & v_{x} & w_{x} \\
\frac{1}{2} & -\frac{1}{2} v & w+\frac{1}{2} v w_{x} v_{x}^{-1}-\frac{1}{2} \psi^{-1} v_{x}^{-2} v_{x x} \\
v_{x} w_{x} \psi & \frac{1}{2} v_{x} u_{x}-v \psi v_{x} w_{x} & \frac{1}{2} \psi w w_{x} v_{x}-\frac{1}{2} \psi^{-1} v_{x}^{-1} \varphi
\end{array}\right) .
\end{aligned}
$$

Operator $\omega_{*}$ reads

$$
\begin{aligned}
\omega_{*}=\left(\begin{array}{c}
\rho \\
\theta \\
\varphi
\end{array}\right)_{*} & =\left(\begin{array}{ccc}
1 & 0 & 0 \\
0 & -v_{x}^{-1} & 0 \\
0 & 0 & \psi v_{x}
\end{array}\right) D_{x}^{2} \\
+ & \left(\begin{array}{ccc}
-u_{x} & -2 w_{x} \psi & -2 \psi v_{x} \\
-\frac{1}{2} & v_{x}^{-2} v_{x x} & v \psi \\
-v_{x} w_{x} \psi & \psi\left(w_{x x}-2 w \psi v_{x} w_{x}-u_{x} w_{x}\right) & -\psi^{2} w v_{x}^{2}-\psi v_{x} u_{x}
\end{array}\right) D_{x} \\
& +\psi^{2}\left(\begin{array}{ccc}
0 & 2 w w_{x} v_{x} & 2 v v_{x} w_{x} \\
0 & c w_{x} & -v^{2} w_{x} \\
0 \psi w^{2} v_{x}^{2} w_{x}-w \psi^{-1} \varphi v_{x}^{2} w_{x}(v w \psi-1)-v \psi^{-1} \varphi
\end{array}\right) .
\end{aligned}
$$

The simplest generalised symmetry of (4.1) is

$$
u_{\tau}=2 v_{x} w_{x} \psi, \quad v_{\tau}=v_{x x}-2 v \psi v_{x} w_{x}+u_{x} v_{x}, \quad w_{\tau}=-w_{x x}+2 w \psi v_{x} w_{x}+u_{x} w_{x}
$$

and does not depend on constant $a$. Hence formula (2.11) can be used to construct the recursion operator for the hierarchy of (4.1). In order to find operator $\mathscr{K}$ we note that the integral $\omega$ satisfies the system

$$
\rho_{\tau}=4 \varphi_{x}, \quad \theta_{\tau}=\theta_{x x}-\left(\theta^{2}\right)_{x}-\frac{1}{2} \rho_{x}, \quad \varphi_{\tau}=-\varphi_{x x}-2(\theta \varphi)_{x} .
$$

The fact that the right hand side of (4.4) is a total derivative alludes to existence of a Hamiltonian operator of the form $A D_{x}$, where $A$ is a constant matrix. This turns out to be true and a symplectic operator required for formula (2.11) is given by

$$
\mathscr{K}=\left(\begin{array}{ccc}
1 & 0 & 0 \\
0 & 0 & -8 \\
0 & -8 & 0
\end{array}\right) D_{x}^{-1} .
$$

The recursion operator obtained from formula (2.11) can be written in a quasi-local form as 


$$
\begin{aligned}
& \mathscr{R}=R_{2} D_{x}^{2}+ R_{1} D_{x}+R_{0}+\left(\begin{array}{c}
u_{x} \\
v_{x} \\
w_{x}
\end{array}\right) D_{x}^{-1} \circ \mathscr{E}(\rho) \\
&-4\left(\begin{array}{c}
1 \\
-v \\
w
\end{array}\right) D_{x}^{-1} \circ \mathscr{E}(\varphi)-4\left(\begin{array}{c}
s_{1} \\
s_{2} \\
s_{3}
\end{array}\right) D_{x}^{-1} \circ \mathscr{E}(\theta),
\end{aligned}
$$

where the matrix coefficients of (4.5) are given by

$$
\begin{aligned}
R_{2}= & \left(\begin{array}{lll}
1 & 0 & 0 \\
0 & 4 & 0 \\
0 & 0 & 4
\end{array}\right), R_{1}=\left(\begin{array}{ccc}
0 & 6 \psi w_{x} & -6 \psi v_{x} \\
3 v_{x} & -8 w_{x} v \psi+4 u_{x} & 0 \\
-3 w_{x} & 0 & -8 v_{x} w \psi-4 u_{x}
\end{array}\right) \\
R_{0}= & u_{x}\left(\begin{array}{ccc}
-u_{x} & 2 \psi w_{x} & 2 \psi v_{x} \\
v_{x} & -4 w_{x} v \psi & -8 \psi v v_{x} \\
w_{x} & 8 \psi w w_{x} & 4 v_{x} w \psi
\end{array}\right)+2 \psi v_{x} w_{x}\left(\begin{array}{ccc}
4 & 5 \psi w & -5 \psi v \\
-4 v-2 \psi v w-3 & 8 \psi v^{2} \\
4 w & 8 \psi w^{2} & -2 \psi v w-3
\end{array}\right) \\
& +2\left(\begin{array}{ccc}
0 & -2 \psi w_{x x} & 2 \psi v_{x x} \\
v_{x x} & 2 \psi v w_{x x} & -\psi\left(v_{x}^{2}+4 v v_{x x}\right) \\
-w_{x x}-\psi\left(4 w w_{x x}+w_{x}^{2}\right) & 2 \psi w v_{x x}
\end{array}\right),
\end{aligned}
$$

where vector $\left(s_{1}, s_{2}, s_{3}\right)^{T}$ is the right-hand side of (4.3).

Remarks. It is known [2] that the hierarchy of higher symmetries of (4.1) is connected with the Yajima-Oikawa hierarchy via a Miura-type transformation. Since the latter is also known to possess a hereditary recursion operator [13], the recursion operator (4.5) is also hereditary [7]. One may wonder if the operators yielded by formulae (2.11) and (2.22) are necessarily hereditary. In general one cannot expect this because the former formula includes an arbitrary linear operator. So, this question reduces to verification of compatibility of Hamiltonian operators of the modified hierarchy.

\section{Acknowledgements}

The author is grateful to anonymous referees for numerous constructive comments which improved this paper.

\section{References}

[1] L.J.F. Broer, Approximate equations for long water waves, Appl. Sci. Res. 31 (1975) 377-395.

[2] D.K. Demskoi, On a class of Liouville-type systems, Theoret. and Math. Phys. 141 (2004), no. 2, 1509-1527.

[3] D.K. Demskoi, V.G. Marikhin, A.G. Meshkov, Lax representations for triplets of two-dimensional scalar fields of chiral type, Theoret. and Math. Phys. 148 (2006), no. 2, 1034-1048.

[4] D.K. Demskoi, A.G. Meshkov, New integrable string-like fields in 1+1 dimensions, in: Proc. 2nd Intl. Conf. Quantum Field Theory and Gravity (July 28-August 2, 1997, Tomsk, Russia, I. L. Bukhbinder and K. E. Osetrin, eds.), Tomsk Pedagogical Univ. Press, Tomsk (1998), pp. 282-285.

[5] D.K. Demskoi, A.G. Meshkov, The Lax representation for a triplet of scalar fields, Theoret. and Math. Phys. 134 (2003), no. 3, 351-364.

[6] D. K. Demskoi, S.Ya. Startsev, On the construction of symmetries from integrals of hyperbolic systems of equations, J. Math. Sci. (N. Y.) 136 (2006), no. 6, 4378-4384. 
[7] A.S. Fokas, B. Fuchssteiner, Bäcklund transformations for hereditary symmetries. Nonlinear Anal. 5 (1981), no. 4, 423-432.

[8] B. Fuchssteiner, A.S. Fokas, Symplectic structures, their Bcklund transformations and hereditary symmetries. Phys. D 4 (1981/82), no. 1, 47-66.

[9] B.S. Getmanov, Higher integrals in two-dimensional models of field theory: Complex scalar fields, in: Group Theory Methods in Physics [in Russian] (Trudy Mezhdunarodnogo Seminara, Zvenigorod, 24-26 Nov., 1982, Vol. 2, M. A. Markov, ed.), Nauka, Moscow (1983), pp. 333-344.

[10] M. Gurses, A. Karasu, and V. V. Sokolov, On construction of recursion operator from Lax representation, J. Math. Phys. 40 (1999) 6473-6490.

[11] D.J. Kaup. A higher-order water-wave equation and the method for solving it, Progr. Theor. Phys. 54 (1975) 396-408.

[12] B.G. Konopelchenko, Nonlinear Integrable Equations. Recursion Operators, Group-Theoretical and Hamiltonian Structures of Soliton Equations, Berlin, Springer, 1987.

[13] Q.P. Liu, Bi-Hamiltonian structures of the coupled AKNS hierarchy and the coupled Yajima-Oikawa hierarchy, J. Math. Phys. 37 (1996), no. 5, 2307-2314.

[14] F. Lund and T. Regge, Phys. Rev. D, 14, 1524 (1976); F. Lund, Solitons and geometry, in: Nonlinear Equations in Physics and Mathematics (NATO Adv. Study Inst. Ser., Ser. C, Vol. 40, A. O. Barut, ed.), Reidel, Dordrecht (1978), pp. 143-175.

[15] M. Marvan, A. Sergyeyev, Recursion operator for the stationary Nizhnik-Veselov-Novikov equation. $J$. Phys. A 36 no. 5 (2003), 87-92

[16] A.G. Meshkov, Hamiltonian and recursion operators for two-dimensional scalar fields. Phys. Lett. A. (1992), 170 (6), 405-408.

[17] A.V. Mikhailov, A.B. Shabat, V.V. Sokolov, The symmetry approach to classification of integrable equations. What is integrability?, 115-184, Springer Ser. Nonlinear Dynam., Springer, Berlin, 1991.

[18] O.I. Mokhov, Symplectic forms on a loop space and Riemannian geometry, Funct. Anal. Appl. 24 (1990), no. 3, 247-249.

[19] P. Olver, Applications of Lie Groups to Differential Equations, Springer, New York (1986).

[20] K. Sawada, T. Kotera. A method for finding n-soliton solutions of the KdV equation and KdV-like equations. Progr. Theor. Phys. 51 (1974) 1355-1367.

[21] V.V. Sokolov, On the symmetries of evolution equations, Russ. Math. Surveys, 43, no. 5, 165-204 (1988).

[22] N. Yajima, M. Oikawa, Formation and Interaction of Sonic-Langmuir Solitons. Inverse Scattering Method, Prog. Theor. Phys. 56 (1976), 1719-1739.

[23] A.V. Zhiber, V.V. Sokolov, Exactly integrable hyperbolic equations of Liouville type, Russ. Math. Surv. 956 (2001) 61-101.

[24] A.V. Zhiber, V.V. Sokolov, S.Ya. Startsev, On nonlinear Darboux-integrable hyperbolic equations, (Russian) Dokl. Akad. Nauk 343 (1995), no. 6, 746-748 